\title{
On the Achievable Bit Rates of DSL Vectoring Techniques in the Presence of Alien Crosstalkers
}

\author{
Amir R. Forouzan ${ }^{1}$, Marc Moonen ${ }^{2,3}$, Michael Timmers ${ }^{4}$, Mamoun Guenach ${ }^{4}$, and Jochen Maes ${ }^{4}$ \\ a.forouzan@eng.ui.ac.ir, marc.moonen@esat.kuleuven.be, \\ \{jochen.maes, michael.timmers\}@alcatel-lucent.com, guenach@ieee.org \\ ${ }^{1}$ Dept. of Electrical Engineering, Faculty of Engineering, University of Isfahan, Isfahan, Iran \\ ${ }^{2}$ ESAT-SCD, KU Leuven, Leuven, Belgium \\ ${ }^{3}$ IBBT-KU Leuven Future Health Dept., KU Leuven, Leuven, Belgium \\ ${ }^{4}$ Bell Labs, Alcatel-Lucent, Antwerp, Belgium
}

\begin{abstract}
Different precoder and equalizer structures have been proposed in literature for vectored digital subscriber lines (DSLs). In this paper, we assess the relative merit of advanced complex precoders and equalizers in practical DSL scenarios. Using extensive computer simulations, the achievable bit rates are investigated in the presence of alien crosstalkers, assuming a beta distribution model for crosstalk couplings. Vectoring in conventional as well as phantom mode (PM) VDSL2 transmission is considered. Vectoring techniques at the access-node (AN) are studied such as the zero-forcing equalizer (ZFE), ZF generalized decision feedback equalizer (ZF-GDFE), linear minimum mean squared error (MMSE), and MMSE-GDFE for upstream (US) scenarios and zero-forcing precoder (ZFP) for downstream (DS) scenarios. Vectoring is also considered at the customer premises (CP) in PM transmission. The results show that when there are no alien crosstalkers, the low-complexity ZFE allows reaching crosstalk-free rates in US direction. However using an MMSEGDFE and if the noise spatial covariance information is assumed static, considerably higher bit rates can be achieved for long loops in the presence of alien crosstalkers. This rate increase comes at the expense of increased delay. In DS direction, the ZFP can achieve crosstalk-free rates in the absence of alien crosstalkers. The results also show that the use of vectoring techniques at the CP in PM transmission does not increase the achievable bit rates in US or DS direction when vectoring is already employed at the AN.
\end{abstract}

Index Terms-Digital subscriber line (DSL), generalized decision feedback equalizer (GDFE), minimum mean squared error (MMSE) equalizer, VDSL2, vectoring, Zero-forcing equalizer (ZFE), Zero-forcing precoder (ZFP).

This research work was carried out in part at the ESAT Laboratory of KU Leuven, in the frame of

Concerted Research Action GOA-MaNet,

The Belgian Programme on Interuniversity Attraction Poles initiated by the Belgian Federal Science Policy Office IUAP P6/04 (DYSCO, 'Dynamical systems, control and optimization', 2007-2011),

KU Leuven Research Council CoE PFV/10/002 'Optimization in Engineering' (OPTEC),

Research Project FWO nr.G.0235.07('Design and evaluation of DSL systems with common mode signal exploitation'), and

IWT Project 'PHANTER: PHysical layer and Access Node TEchnology Revolutions: enabling the next generation broadband network'.

The scientific responsibility is assumed by its authors.

\section{INTRODUCTION}

Digital subscriber line (DSL) technology provides a costefficient broadband access solution for homes and small businesses. However, the achievable bit rates on DSL loops can drop significantly when more than one loop uses the same telephone cable binde due to the mutual crosstalk between them. Vectoring is a promising solution to counteract crosstalk and potentially retain crosstalk-free rates in DSL [1]-[4]. Vectoring is usually implemented at the access node (AN), where the loops' ends are co-located and the signals of all users are available for joint processing. However $3 \times 3$ vectoring techniques can also be considered at the customer premises (CP) in phantom mode (PM) transmission. PM transmission applies to systems in which transmission takes place over a quad wire or two pairs of twisted wire pairs with co-located ends at both sides providing three balanced transmission modes (instead of two), i.e., two differential modes and one phantom mode [5]. Transmission over the unbalanced common mode comprising the four wires as forward conductors and the ground as return is not considered.

Several vectoring schemes are available for DSL. The zeroforcing equalizer (ZFE) [2] and zero-forcing generalized decision feedback equalizer (ZF-GDFE) [1], linear minimum mean squared error equalizer (MMSE) [6], and MMSE-GDFE [7], [8] are implemented at the receiver side (e.g., at the AN in US direction) and the ZF precoder (ZFP) [3] and ZF TomlinsonHarashima precoder (ZF-THP) [1] are implemented at the transmitter side (e.g., at the AN in DS direction). From an implementation complexity perspective, the ZFE and ZFP are the most effective techniques. The ZFE and ZFP require the direct and crosstalk channel information for the coordinated users only. The linear MMSE and MMSE-GDFE require noise spatial covariance information as well. This means 1) a higher implementation complexity and 2) a need for an additional signal-to-noise ratio margin (SNRM). The latter is considered to tackle abrupt changes in the noise spatial covariance information when uncontrolled lines activate or deactivate. The ZF-GDFE and MMSE-GDFE again have a 
higher implementation complexity than the ZFE and linear MMSE, respectively. Similarly, the ZF-THP has a higher implementation complexity than ZFP. In the ZF-GDFE and MMSE-GDFE signals are decoded sequentially. In presence of channel coding, this may result in a much longer decoding delay for the signals that are decoded later in the sequence [1].

Generally speaking, the ZF-GDFE, linear MMSE, and MMSE-GDFE provide a superior performance than the ZFE and similarly the ZF-THP has a superior performance compared the ZFP. Having the trade-off between the implementation complexity and performance in mind, it is interesting to quantify the performance gains of these schemes w.r.t. the performance of the ZFE and ZFP under realistic DSL channel conditions.

It has been shown that the ZFE and ZFP can achieve crosstalk-free rates when all users are coordinated assuming a $1 \%$ worst-case crosstalk channel model [2], [3]. In practice, the crosstalk couplings are random variables and there may be uncoordinated users in the cable binder because of loop unbundling or implementation complexity constraints [9]. Our aim is to study the achievable bit rates and compare the performance of the vectoring techniques under more realistic conditions by taking into account the channel randomness and possible presence of uncoordinated or alien users. We study vectoring in US and DS directions. For US, we investigate the performance of ZFE, ZF-GDFE, linear MMSE, and MMSEGDFE at the AN and ZFP at the $\mathrm{CP}$ when PM transmission is enabled. For DS, we investigate the ZFP at the AN and MMSE and MMSE-GDFE at the CP when PM transmission is enabled. The crosstalk channel is simulated using a beta distribution model for crosstalk amplitude proposed in [10].

The paper is organized as follows. In Sec. II, we describe the investigated scenarios and the channel model. In Sec. III, we explain the simulations setup for the scenarios in Sec. II. Simulation results are provided in Sec. IV. Finally the conclusion are given in Sec. V.

\section{SCENARIO DESCRIPTION}

Two scenarios have been investigated. In Scen. A, all users transmit in differential transmission mode over a single twisted wire pair. In Scen. B, the users transmit in phantom mode (PM) over a quad or two equi-length twisted wire pairs with co-located ends at both sides.

\section{A. Scenario A: Differential Transmission Mode}

We assume a total of $N_{\text {tot }}$ VDSL2 loops. Each loop corresponds to a distinct user. The first $N_{\text {coord }}$ loops are the coordinated loops and the rest of the loops are uncoordinated and act as alien crosstalkers to the first group. The maximum transmit power spectral density (PSD) of the users is determined by the VDSL2 997E17 regulatory PSD mask level [11], [12]. Upstream power back-off (UPBO) [11], [12] is also enabled on all loops. The loop lengths are chosen randomly with uniform distribution over $\{300,350, \ldots, 1000\} \mathrm{m}$.
The direct channel transfer function of the loops (denoted by $T_{\mathrm{f}}(\ell)$ for a loop with length $\ell$ ) is calculated by the 26 AWGN cable model. The crosstalk channel transfer function from transmitter $m$ to receiver $n$ in US direction is calculated by the beta distribution model as follows

$$
h_{n m}^{\mathrm{US}}=\beta_{n m} e^{j \phi_{n m}} f \sqrt{k_{\mathrm{f}} \min \left\{\ell_{n}, \ell_{m}\right\}} T_{\mathrm{f}}\left(\ell_{m}\right),
$$

where $\phi_{n m}$ is a random variable with uniform distribution over $[0,2 \pi)$ modeling the crosstalk channel phase, $k_{\mathrm{f}}=2.539 \times$ $10^{-20}$ is the FEXT coupling constant, $f$ is frequency in Hertz, and $\ell_{n}$ and $\ell_{m}$ are the loop lengths in meter. The parameter $\beta_{n m}$ is the offset of the crosstalk channel w.r.t. the standard $1 \%$ worst-case crosstalk channel. In our simulations, $\beta_{n m}$ is set to the element located at row $n$ and column $m$ of the beta offset matrix of [13]. The resulting crosstalk couplings follow the beta crosstalk model obtained by practical measurements in [10]. Vectoring is merely applied at the AN in Scen. A. The vectoring techniques which are investigated include the ZFE, ZF-GDFE, linear MMSE, and MMSE-GDFE.

The MMSE-GDFE is implemented in two ways w.r.t. the decoding order of the users/loops. In the first implementation, the decoding order of the loops is simply determined by their index where the last coordinated loop is decoded first and the first coordinated loop is decoded last. When decoding each loop, the GDFE structure removes the interference of the loops that are already decoded. Long loops reach much smaller rates than short loops due to the higher loop loss and the near-far effect ${ }^{1}$. Therefore, in the second implementation, the decoding order is based on the loop length, where the shortest loop is decoded first and the longest loop is decoded last. The longer loops potentially reach a higher rate in the second implementation compared to what they reach in the first implementation.

\section{B. Scenario B: Phantom Mode}

Scenario B models PM transmission. We assume $N_{\text {tot }}$ VDSL2 loops of $N_{\mathrm{CP}}=\left\lceil N_{\text {tot }} / 3\right\rceil$ users each transmitting over two twisted wire pairs (or a quad wire) providing three transmission modes, i.e., two differential modes and one common mode. This means that there are (approximately) a total of $2 N_{\mathrm{CP}}$ wire-pairs and $3 N_{\mathrm{CP}}$ operating VDSL2 loops ${ }^{2}$. We assume that the first $N$ users, corresponding to the first $N_{\text {coord }}=3 N$ transmission modes or VDSL2 loops, are coordinated. The remaining $N_{\text {tot }}-3 N$ VDSL2 loops act as alien crosstalkers. The direct channel transfer functions for all transmission modes (both differential and common modes) are calculated using the 26 AWG cable model [14]. The crosstalk channel transfer function of the common mode to/from the differential mode of the same quad is modeled by (1) with $\beta_{n m}$ set to $0 \mathrm{~dB}$, i.e., the standard $1 \%$ worst-case model with a random phase uniformly distributed over $[0,2 \pi)^{3}$. All other crosstalk transfer functions are modeled by (1) as in

\footnotetext{
${ }^{1}$ Near-far effect is partially or fully compensated using UPBO

${ }^{2}$ When $N_{\text {tot }}$ is not a multiple of 3 , the last one or two transmission modes are assumed to (be equi-length and) transmit in differential mode

${ }^{3}$ This assumption is made based on practical measurement results.
} 


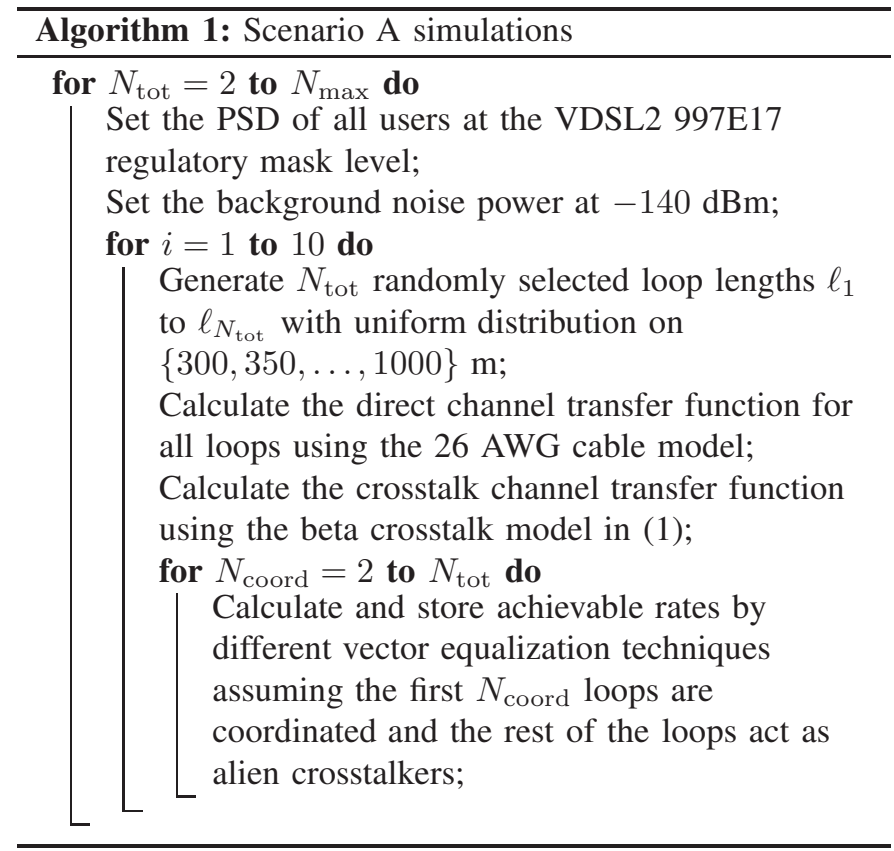

Scen. A. Scenario B is simulated in both US and DS directions. The beta distribution crosstalk model is given in (1) for US direction. The beta model for DS direction is

$$
h_{n m}^{\mathrm{DS}}=\beta_{n m} e^{j \phi_{n m}} f \sqrt{k_{\mathrm{f}} \min \left\{\ell_{n}, \ell_{m}\right\}} T_{\mathrm{f}}\left(\ell_{n}\right) .
$$

The transmit PSDs for all transmission modes are set equal to the VDSL2 997E17 regulatory PSD mask level [11], [12] with UPBO enabled in US direction. Background noise power is set to $-140 \mathrm{dBm}$.

For Scen. B we consider $N_{\text {coord }} \times N_{\text {coord }}$ vectoring at the $\mathrm{AN}$ and $3 \times 3$ vectoring at the CPs. In US direction, we consider four different vectoring techniques, namely, ZFE, ZFGDFE, linear MMSE, and MMSE-GDFE, at the AN and $3 \times 3$ ZFP at the CPs. In DS direction, we consider $N_{\text {coord }} \times N_{\text {coord }}$ $\mathrm{ZFP}$ at the AN and $3 \times 3$ linear MMSE or MMSE-GDFE at the CPs.

\section{Simulation Setup}

ZFE, ZF-GDFE, linear MMSE, and MMSE-GDFE have been simulated for Scen. A for various values of $N_{\text {tot }}$ and $N_{\text {coord }}$. A pseudo-code for the simulations is presented in Alg. 1. Two nested loops set the values of $N_{\text {tot }}$ and $N_{\text {coord }}$ from 2 to $N_{\max }$ and 2 to $N_{\text {tot }}$, respectively, where $N_{\max }$ is a constant. For each $\left(N_{\text {tot }}, N_{\text {coord }}\right)$ pair, simulations are repeated ten times using different sets of loop lengths.

The achievable rates are then averaged per loop length to obtain an estimation of the ergodic achievable rates [15] of these equalization techniques. Note that through the averaging, all information on the spread on achievable rates is ignored. Since the average number of loops of each loop length is proportional to $N_{\text {coord }}$ for each $\left(N_{\text {tot }}, N_{\text {coord }}\right)$ pair, more weight is given to scenarios with low number of aliens.

The performance of different vectoring techniques is evaluated for Scen. B in a similar fashion. Since we consider

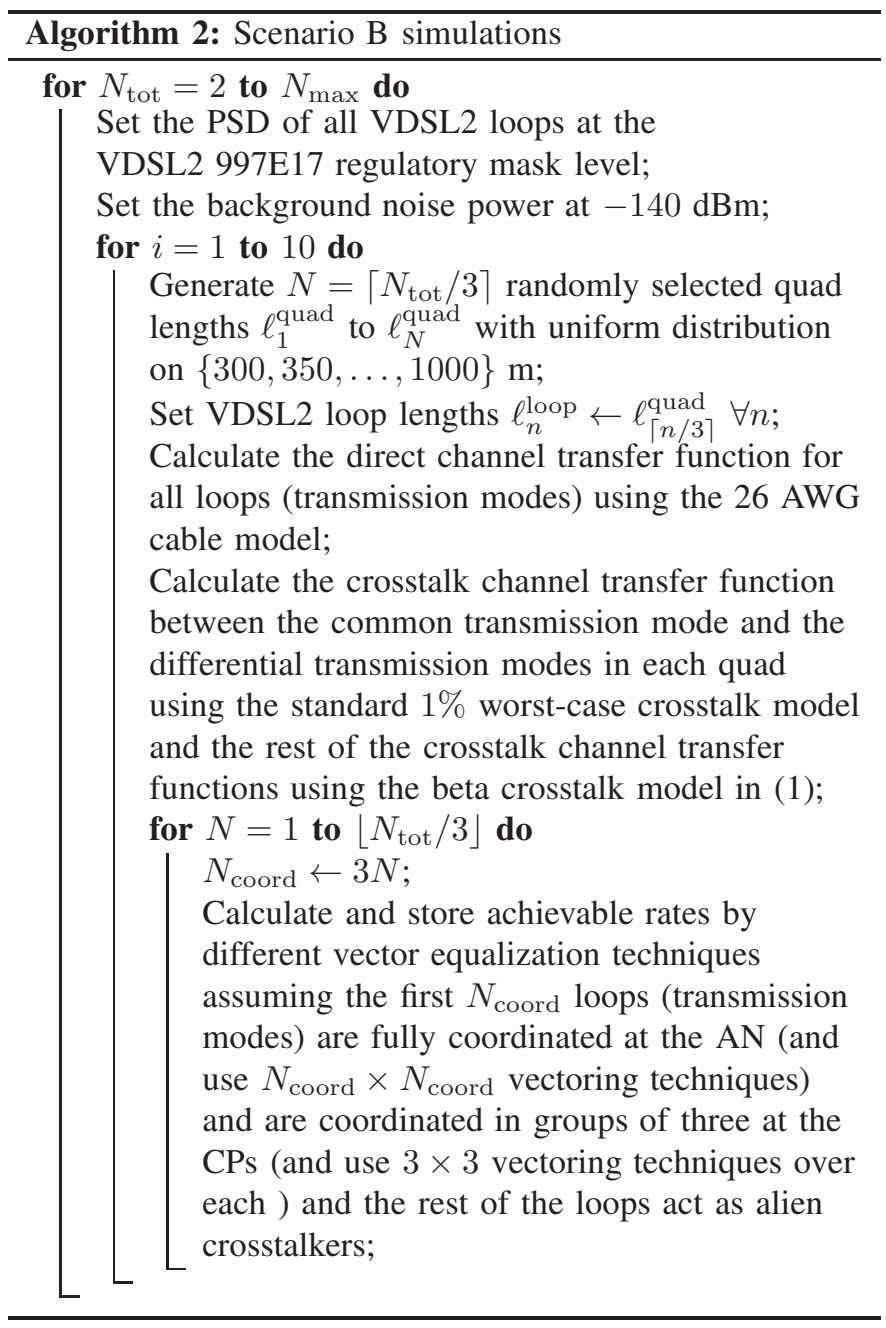

Phantom Mode transmission in Scen. B, the loop lengths for every three transmission modes are the same. Therefore, with $N_{\text {tot }}$ transmission modes, we generate $\left\lceil N_{\text {tot }} / 3\right\rceil$ random loop lengths. Then we generate the direct and crosstalk channel transfer function, using the rules described in Sec. II-B. Simulations are carried out for both US and DS direction for Scen. B. In each case, $N_{\text {coord }} \times N_{\text {coord }}$ vectoring is available at the AN, and $3 \times 3$ vectoring is available at the CPs. A pseudo-code for Scen. B simulations is presented in Alg. 2.

\section{Simulation Results}

Simulation results for Scenario A in upstream direction are presented in Figures 1 to 3. The average rates achievable by different vectoring techniques when all loops are coordinated and no alien crosstalkers are present are plotted versus loop length in Figure 1. As it can be seen, all vectoring techniques (including the ZFE which is the least complex among them) can reach the crosstalk-free rates (labeled "No xtalk"). This is in agreement with the results in [2]. The average rates achievable in absence of any vectoring technique are labeled "SSM" (Static Spectrum Management).

Figures 2 to 3 show the simulation results for Scen. A 


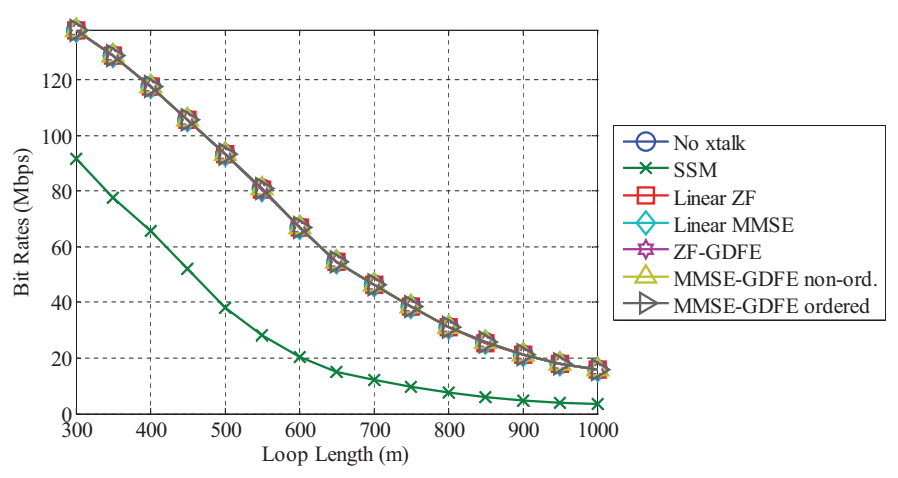

Fig. 1. US average bit-rates vs. loop length for different vectoring techniques for Scen. A in the absence of alien crosstalkers for $N_{\max }=73$.

in the presence of alien crosstalkers. The average achievable rates are plotted vs. loop lengths in Fig. 2. Figure 3 shows the obtained rates averaged over all loop lengths. Two sets of results are presented for the MMSE-GDFE. The results labeled "MMSE-GDFE non-ord." refer to the case where the decoding order is set according to the users index and the results labeled "MMSE-GDFE ordered" refer to the case where the decoding order is set according to the loop lengths. None of the schemes achieve the crosstalk free rates in the presence of alien crosstalkers. More importantly, the ZFE still achieves the same rates as the ZF-GDFE, however, they do not perform as well as the linear MMSE and MMSE-GDFE. The MMSE-GDFE achieves considerably higher average rates than the ZFE, ZF-GDFE, and linear MMSE for long loop lengths. Particularly, when the decoding order is set according to the loop lengths, the gains are much higher for long loops. However, the MMSE-GDFE results in smaller rates for short loops when the decoding order is set according to the loop lengths. Overall, as it can be seen in Fig. 3, the average achievable rates by the MMSE-GDFE does not change by changing the decoding order. This can be justified by noting that the MMSE-GDFE reaches the sum capacity of the multiple-access channel regardless of the order of decoding [16], [17].

A slight rate increase is observed for linear MMSE compared to linear ZF. This increase cannot be guaranteed in practice due to uncontrolled and abrupt changes in the noise spatial covariance information. Taking into account a SNRM is required to ensure system stability, the curve for MMSE will shift downward, resulting in ZFP to outperform MMSE in practice.

Figure 4 and 5 illustrate the simulation results on average bit rates per transmission mode by different schemes in Scen. $\mathrm{B}^{4}$. To assess the effect of $3 \times 3$ Phantom Mode vectoring at the CPs, two groups of simulations have been carried out. In the first group, vectoring is available only at the AN. Techniques that belong to this group are the ZFE, linear MMSE, ZF-GDFE, MMSE-GDFE, and MMSE-GDFE

\footnotetext{
${ }^{4}$ Each user transmits over three transmission modes. Therefore the achievable rates by the users is three times those reported in Figs. 4 and 5
}

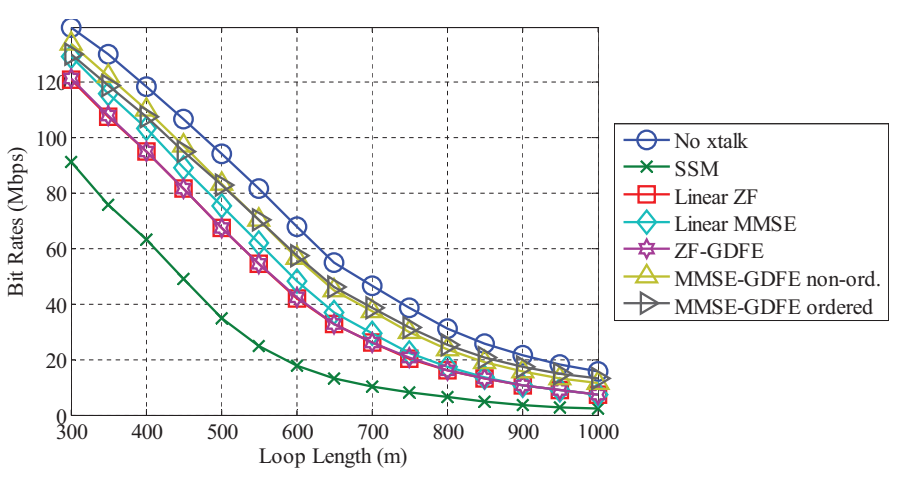

Fig. 2. US average bit-rates vs. loop length for different vectoring techniques for Scen. A in the presence of alien crosstalkers for $N_{\max }=73$.

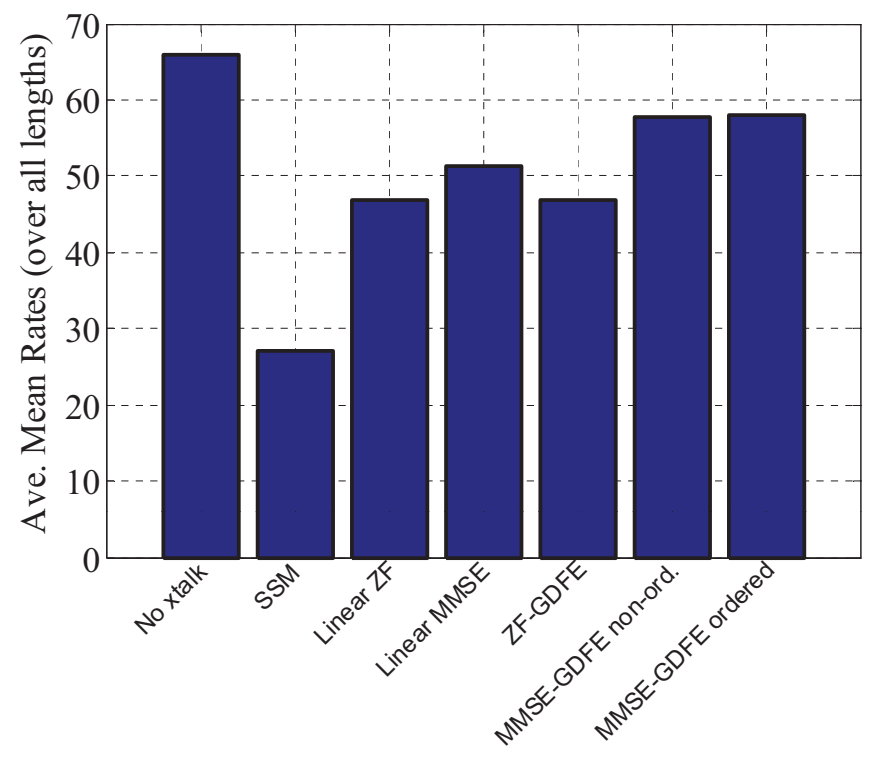

Fig. 3. US achievable bit-rates averaged over all loop lengths for different vectoring techniques for Scen. A in the presence of alien crosstalkers for $N_{\max }=73$.

with decoding order according to the loop lengths and are labeled "linear ZFE", "linear MMSE", "ZF-GDFE", "MMSEGDFE", and "MMSE-GDFE(ordered)", respectively. All of these vectoring techniques are implemented at the AN which is the receiver side in US direction. In the second group, ZFP is used at the CPs which is the transmitter side. At the $\mathrm{AN}$, one of the following vectoring techniques has been used: ZFE, linear MMSE, and MMSE-GDFE. These techniques are labeled "ZFE + linear ZFP @ TX", "linear MMSE + ZFP @ TX', and "MMSE-GDFE + ZFP @ TX", respectively.

Comparing the results of the first group to those of the second group, we see that $3 \times 3$ vectoring at the CPs does not have any effect on the achievable rates when vectoring is available at the AN. Moreover, a sensible drop in the achievable bit rates of SSM and the vectoring schemes can be seen, if we compare the results to the achievable bit rates in Scen. A. This can be attributed to the stronger crosstalk couplings in Phantom Mode transmission. 


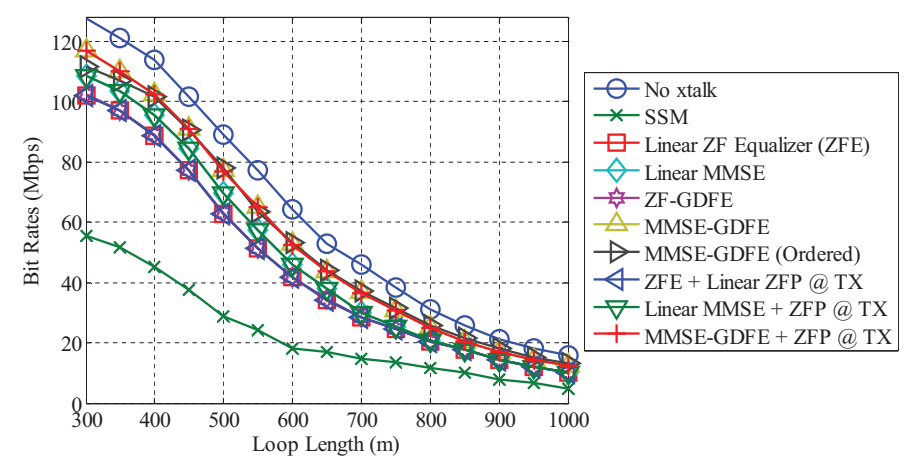

Fig. 4. US average bit-rates vs. loop length for different vectoring techniques for Scen. B in the presence of alien crosstalkers for $N_{\max }=36$.

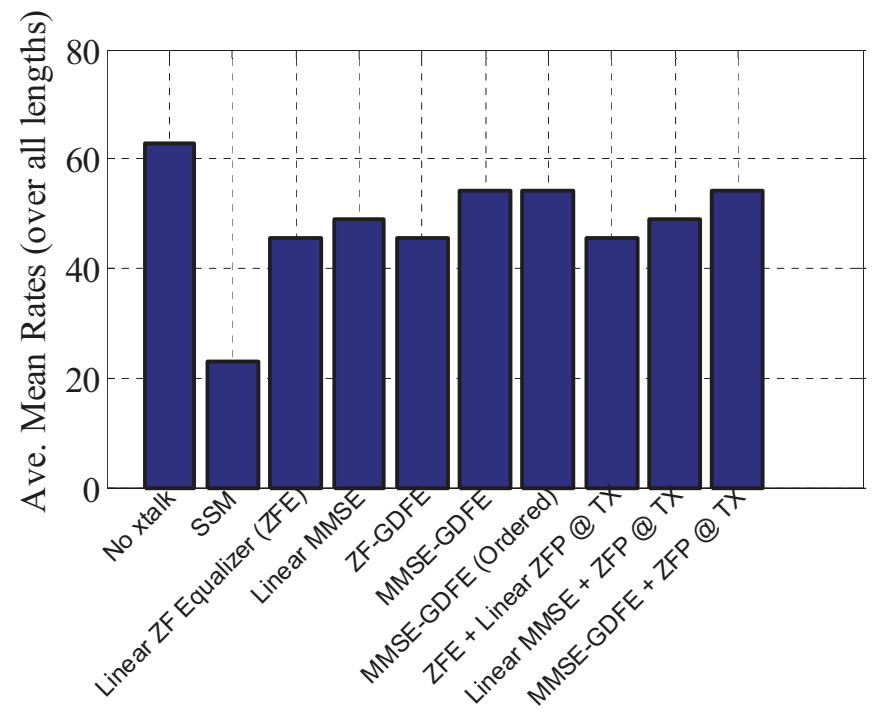

Fig. 5. US achievable bit-rates averaged over all loop lengths for different vectoring techniques for Scen. B in the presence of alien crosstalkers for $N_{\max }=36$.

Finally, Figs. 6 to 8 show simulation results for Scen. B in downstream direction. We have studied the effect of $3 \times 3$ Phantom Mode vectoring in this case as well. In DS direction, the transmitters are located at the $\mathrm{AN}$ and the receivers are located at the CPs. Therefore, $N_{\text {coord }} \times N_{\text {coord }}$ precoding techniques may be used at the AN and assuming PM transmission, $3 \times 3$ equalization techniques may be used at the CPs. In this work, we have simulated the ZFP at the AN with or without linear MMSE or MMSE-GDFE a the CPs. The techniques are labeled "ZFP", "ZFP + linear MMSE", and "ZFP + MMSE-GDFE", respectively. Figure 6 shows the simulation results when all users are coordinated, i.e., no alien crosstalkers are present. As it can be seen, all schemes can reach the crosstalk free rates. Therefore, when all users are coordinated, PM vectoring is not needed at the CPs. In fact, the (near-) optimallity of the ZFP in this case has already been shown in [3].

Figures 7 and 8 present the simulation results in which alien crosstalkers are present. As it can be seen, none of the

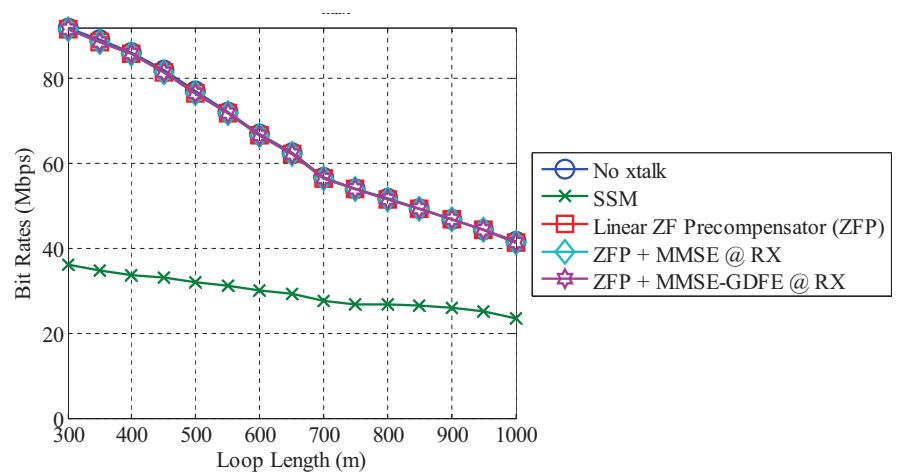

Fig. 6. DS average bit-rates vs. loop length for different vectoring techniques for Scen. B in the absence of alien crosstalkers for $N_{\max }=89$.

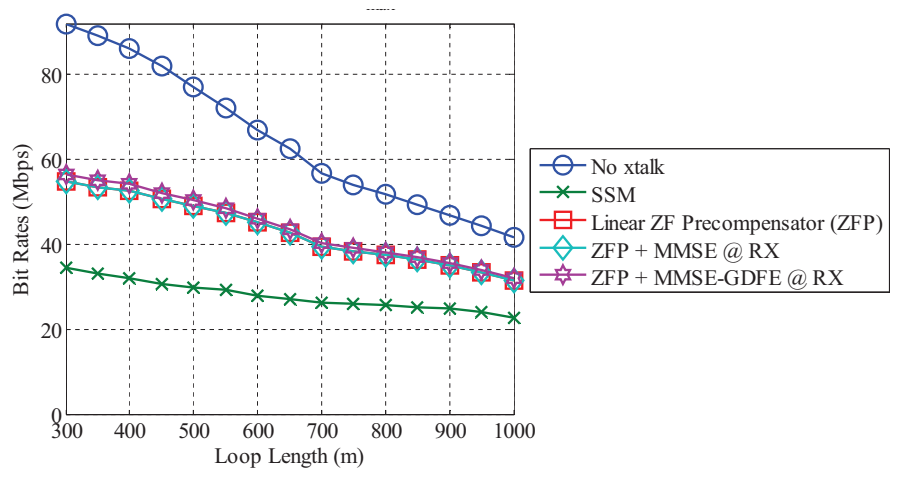

Fig. 7. DS average bit-rates vs. loop length for different vectoring techniques for Scen. B in the presence of alien crosstalkers for $N_{\max }=89$.

techniques is able to reach crosstalk free rates in this case. Moreover, using PM vectoring at the receiver side does not increase the achievable rates in general. In fact, compared to the low-complex ZFP, the achievable rates do not increase when $3 \times 3$ linear MMSE equalizers are used at the $\mathrm{CP}$ and they increase slightly when $3 \times 3$ MMSE-GDFE equalizers are used.

\section{Summary AND CONCLUSION}

In this paper, we have presented simulation results of DSL vectoring schemes in two scenarios. In Scen. A, we have considered conventional differential mode DSL transmission and in Scen. B we have considered phantom mode transmission. We have studied the average rates in US direction for Scen. A using equalization techniques at the $\mathrm{AN}$, and the average rates in US and DS directions for Scen. B using vectoring schemes at the $\mathrm{AN}$ as well as CPs. Through the rate averaging the spread on achievable rates is ignored and the reported average rates are dominated by scenarios with low number of aliens. Our results for different cases are summarized as follows:

Scenario A (US direction):

- The ZFE is optimal when noise is not spatially colored (i.e. in the absence of alien crosstalkers).

- The MMSE-GDFE performs considerably better than ZFE in the presence of alien crosstalkers. 


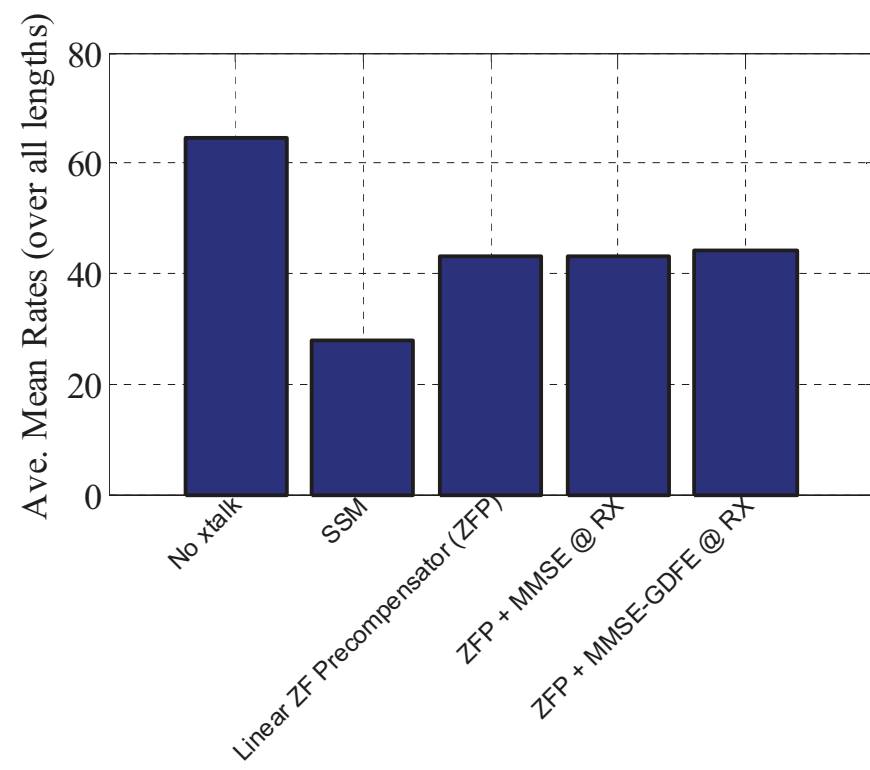

Fig. 8. DS achievable bit-rates averaged over all loop lengths for different vectoring techniques for Scen. B in the presence of alien crosstalkers for $N_{\max }=89$.

- By setting the decoding order in the MMSE-GDFE according to the loop lengths, higher rates for long lowrate loops can be achieved.

Scenario B (US direction):

- The results follow the same trend as those in Scen. A in US direction.

- When vectoring is used at the AN, utilization of $3 \times 3$ ZFPs at the CPs has no significant effect on the achievable rates regardless of the vectoring technique at the AN.

Scenario $B$ (DS direction):

- Crosstalk free rates can be achieved with ZFP in the absence of alien crosstalkers (90 to $150 \%$ increase in rates).

- In the presence of alien crosstalkers use of MMSE or MMSE-GDFE at the RX side does not help when ZFP is already used at the transmitter side.

In conclusion, linear MMSE shows some benefit over ZFE under static noise spatial covariance for some loop lengths in presence of alien crosstalkers. However, maintaining the noise spatial covariance information can be impractical when alien crosstalkers are not under control. MMSE-GDFE provides considerably better data rates compared to ZFE. However, it requires the noise spectral covariance information as well. Besides, the GDFE decoding delay makes it somehow unattractive from the implementation and application points of view. When multiple loops are terminated at a single CP, two-sided signal coordination at both the $\mathrm{AN}$ and $\mathrm{CP}$ does not provide notable improvements compared to signal coordination at the AN only, even in the presence of alien crosstalkers.

\section{REFERENCES}

[1] G. Ginis and J. M. Cioffi, "Vectored transmission for digital subscriber line systems," IEEE J. Select. Areas Commun., vol. 20, no. 5, pp. 1085 1104, Jun. 2002.

[2] R. Cendrillon, G. Ginis, E. Van den Bogaert, and M. Moonen, "A near-optimal linear crosstalk canceler for upstream VDSL," IEEE Trans. Signal Processing, vol. 54, no. 8, pp. 3136-3146, Aug. 2006.

[3] _ "A near-optimal linear crosstalk precoder for downstream VDSL," IEEE Trans. Commun., vol. 55, no. 5, pp. 860-863, May 2007.

[4] V. Oksman, H. Schenk, A. Clausen, J. Cioffi, M. Mohseni, G. Ginis, C. Nuzman, J. Maes, M. Peeters, K. Fisher, and P.-E. Eriksson, "The ITU-T's new G.vector standard proliferates $100 \mathrm{Mb} / \mathrm{s}$ DSL,' IEEE Commun. Mag., vol. 48, no. 10, pp. 140-148, Oct. 2010.

[5] W. Foubert, C. Neus, L. Van Biesen, and Y. Rolain, "Exploiting the phantom-mode signal in DSL applications," IEEE Trans. Instrumentation \& Measurement, vol. PP, no. 99, pp. 1-7, 2011.

[6] I. Wahibi, M. Ouzzif, J. L. Masson, and S. Saoudi, "Crosstalk cancellation in upstream coordinated dsl using an iterative mmse receiver," in IEEE Int'l. Conf. on Commun., ICC'09, Dresden, Germany, Jun. 2009.

[7] P. Tsiaflakis, J. Vangorp, J. Verlinden, and M. Moonen, "Multiple access channel optimal spectrum balancing for upstream DSL transmission,' IEEE Commun. Lett., vol. 11, no. 4, pp. 398-300, Apr. 2007.

[8] A. R. Forouzan, M. Moonen, J. Maes, and M. Guenach, "Efficient calculation of decoding order in non-ideal DSL multiple-access channels," in 17th IEEE Symp. on Commun. \& Veh. Technol. in the Benelux, SCVT'10, Enschede, The Netherlands, Nov. 2010, pp. 1-6.

[9] M. Guenach, J. Meas, M. Timmers, O. Lamparter, J. Bischoff, and M. Peeters, "Vectoring in DSL systems: Practices and challenges," in IEEE Global Telecom. Conf., GLOBECOM'11, Houston, TX, Dec. 2011.

[10] J. Maes, M. Guenach, and M. Peeters, "Statistical MIMO channel model for gain quantification of DSL crosstalk mitigation techniques," in IEEE Int'l. Conf. on Commun., ICC'09, Dresden, Germany, Jun. 2009, pp. 15.

[11] ITU-T G.993.2, "Very high speed digital subscriber line transceivers 2 (VDSL2)," Geneva, Switzerland, Feb. 2006.

[12] ITU-T G.993.2; Amendment 1, "Very high speed digital subscriber line transceivers 2 (VDSL2)," Geneva, Switzerland, Apr. 2007.

[13] NIPP-NAI-2007-010R2, "100x100 FEXT coupling matrix," AT\&T, Adtran \& Conexant, Nashvile, USA, Feb. 2007.

[14] P. Golden, H. Dedieu, and K. Jacobsen, Fundamentals of DSL Technology. Boca Raton, FL: Auerbach Publications, 2006.

[15] S. Huberman and T. Le-Ngoc, "Ergodic capacity of a DSL binder channel," in IEEE Global Telecom. Conf., GLOBECOM'11, Houston, TX, Dec. 2011.

[16] B. Rimoldi and R. Urbanke, "A rate-splitting approach to the Gaussian multiple-access channel," IEEE Trans. Inform. Theory, vol. 42, no. 2, pp. 364-375, Mar. 1996.

[17] D. N. C. Tse and S. V. Hanly, "Multiaccess fading channels. I. Polymatroid structure, optimal resource allocation and throughput capacities,' IEEE Trans. Inform. Theory, vol. 44, no. 7, pp. 2796-2815, Nov. 1998. 